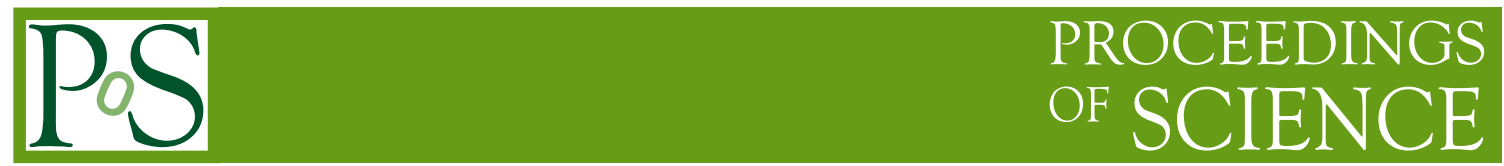

\title{
$\alpha_{s}$ in 2016 from the (revised) ALEPH data for $\tau$ decay
}

\section{Diogo Boito}

Instituto de Física de São Carlos, Universidade de São Paulo CP 369, 13570-970, São Carlos, SP, Brazil

\section{Maarten Golterman}

Dept. of Physics and Astronomy, San Francisco State University

San Francisco, CA 94132, USA

\section{Kim Maltman}

Dept. of Mathematics and Statistics, York Univ.

Toronto, ON Canada M3J 1P3

CSSM, University of Adelaide

Adelaide, SA 5005, Australia

\section{Santiago Peris*}

Dept. of Physics and IFAE-BIST, Univ. Autònoma de Barcelona E-08193 Bellaterra, Barcelona, Spain

E-mail: perisdifae.es

\begin{abstract}
We summarize a comparison of the two strategies which are currently available in the literature for determining the value of $\alpha_{s}\left(m_{\tau}\right)$. We will refer to these as the truncated Operator Product Expansion model and the Duality Violation model. After describing the main features of both approaches, we explain why the former fails to pass crucial tests. The latter, on the other hand, passes all the tests known up to date and, therefore, should be currently considered the only reliable method.
\end{abstract}

38th International Conference on High Energy Physics 3-10 August 2016

Chicago, USA

${ }^{*}$ Speaker. 
The $\tau$ is the only lepton capable of decaying into hadrons. This makes it a potentially clean experimental environment to study QCD and, particularly, to determine the strong coupling $\alpha_{s}$. Furthermore, its mass $m_{\tau} \simeq 1.8 \mathrm{GeV}$ is both large enough to allow the use of perturbation theory (supplemented by nonperturbative corrections), and small enough to potentially compete in precision with other determinations of $\alpha_{s}$, carried out at much higher energies such as the $Z$ mass. This is because the relative error in $\alpha_{s}$ at the $Z$ mass, $\varepsilon\left(M_{Z}\right)$, gets squeezed from its value at the $\tau$ scale by $\varepsilon\left(M_{Z}\right) \simeq \varepsilon\left(m_{\tau}\right)\left(\alpha_{s}\left(M_{Z}\right) / \alpha_{s}\left(m_{\tau}\right)\right)$. Since no lunch is free, however, the price to pay at the $\tau$ scale is that nonperturbative corrections are non-negligible, and it is absolutely essential to bring them under good theoretical control. Otherwise, the determination of $\alpha_{s}$ becomes unreliable.

In 1992, Braaten, Narison and Pich [四], followed by LeDiberder and Pich [వ], building on previous work [B] -[四], showed how Perturbation Theory (PT) and the Operator Product Expansion (OPE) could potentially be used to produce a precise determination of $\alpha_{s}\left(m_{\tau}\right)$. This method has been the traditional choice until recently, and has been applied in a series of analyses based on the hadronic data collected in the OPAL [ए]] and the ALEPH [ए], [13] experiments. ${ }^{1}$

The error originally estimated for $\alpha_{s}\left(m_{\tau}\right)$ in 1992 ranged from $10 \%-30 \%$ [四; that currently claimed in Ref. [13]] is roughly 2\% (in CIPT). Of course, most of the improvement in precision is due to the more accurate data presently available. This improvement in experimental precision, however, should also be accompanied by an improvement in the theoretical description used to analyze these data. The theoretical framework used in these traditional analyses has, however, remained largely the unmodified version of the original one from Ref. [వ]].

This motivated us in a series of papers [[ए] [[ㅁ] to take a fresh look at the assumptions underlying these traditional determinations of $\alpha_{s}\left(m_{\tau}\right)$. The outcome of these investigations is a new method to analyze the hadronic $\tau$ data. This led to a new, significantly lower result for $\alpha_{s}\left(m_{\tau}\right)$ in Ref. []] ]. This calls, of course, for a closer investigation.

Here we would like to point out some of the main differences between the two methods. In disagreement with Ref. [22] our conclusion will be that the method discussed in Ref. [22] has fundamental flaws and, therefore, that the method of Ref. [D] is the only reliable one currently available. Due to space limitations, only a brief summary of our results will be presented. The reader is referred to Ref. [ए23] for a more detailed account.

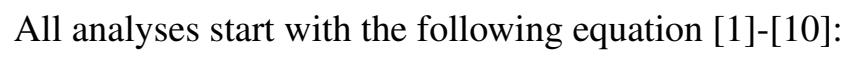

$$
\begin{array}{rl}
\frac{1}{s_{0}} \int_{0}^{s_{0}} & d s w\left(s / s_{0}\right) \rho_{V / A}^{(1+0)}(s) \\
\quad & =-\frac{1}{2 \pi i s_{0}} \oint_{|s|=s_{0}} d s w\left(s / s_{0}\right) \Pi_{\mathrm{OPE}, V / A}^{(1+0)}(s)-\frac{1}{s_{0}} \int_{s_{0}}^{\infty} d s w\left(s / s_{0}\right) \frac{1}{\pi} \operatorname{Im} \Delta_{V / A}(s)
\end{array}
$$

where $w(x)$ is a convenient polynomial, $\rho_{V / A}^{(1+0)}(s)$ is the $1+0$ spin combination for the vector/axial vector spectral function, $\Pi_{\mathrm{OPE}, V / A}^{(1+0)}(s)$ is the OPE approximation to the corresponding correlator, and $\operatorname{Im} \Delta_{V / A}(s)$ contains the so-called Duality Violations (DVs) [24]. This DV term is supposed to compensate for the lack of convergence of the OPE on the Minkowski axis and, in more physical terms, is responsible for the mismatch between the short-distance quark-gluon description and

\footnotetext{
${ }^{1}$ The latest ALEPH data were corrected in Ref. [[13] after an error in its covariance matrices was detected in Ref. [14]].
} 
the long-distance hadronic description of the correlator $\Pi_{\mathrm{OPE}, V / A}^{(1+0)}(s)$. This correlator contains the perturbative series (and the nonperturbative condensates) and depends on $\alpha_{s}$, whereas $\rho_{V / A}^{(1+0)}(s)$ contains the experimental data. The maximum value of $s_{0}$ is $m_{\tau}^{2}$.

It is clear that Eq. (II) can only determine $\alpha_{s}$ after one assumes something about $\operatorname{Im} \Delta_{V / A}(s)$.

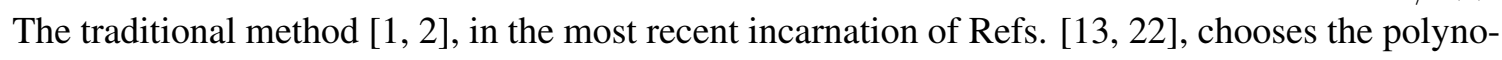
mial $w\left(s / s_{0}\right)$ from the set $w_{k \ell}(x)=(1-x)^{k+2} x^{\ell}(1+2 x)$, with $(k \ell) \in\{(00),(10),(11),(12),(13)\}$. The hope is that, because these polynomials have a zero on the Minkowski axis of degree two or three, the contribution from the OPE on the region of the contour $|s|=s_{0}$ touching this axis, where the OPE is not valid, will be suppressed enough not to require any compensating term in the form of $\operatorname{Im} \Delta_{V / A}(s)$. To make this possibility more likely, $s_{0}$ is chosen equal to $m_{\tau}^{2}$, since the OPE works better at higher scales. With all these choices, the net result is that $\operatorname{Im} \Delta_{V / A}(s)$ is assumed to be zero.

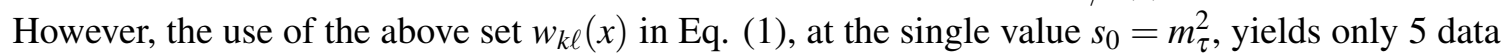
points, whereas these polynomials contain powers of $s$ all the way up to $s^{7}$. Cauchy's theorem then tells us that all condensates, $C_{D}$, from the OPE will contribute to Eq. (II) for $D=4,6,8,10,12,14$ and 16. Together with $\alpha_{s}$, that makes 8 unknowns for 5 data points. In order to be able to do a fit with one degree of freedom, the condensates with $D=10,12,14$ and 16 are set to zero by hand. The bottom line is that one has to make two assumptions for the traditional method to be operational: negligible DVs and a truncation of higher-dimension OPE terms. These two assumptions are actually related [25].

In contrast, the method of Ref. [2] chooses the following explicit parametrization for $\rho_{V / A}^{\mathrm{DV}}(s)$ $\equiv \frac{1}{\pi} \operatorname{Im} \Delta_{V / A}(s)$ :

$$
\rho_{V / A}^{\mathrm{DV}}(s)=e^{-\delta_{V / A}-\gamma_{V / A} s} \sin \left(\alpha_{V / A}+\beta_{V / A} s\right), \quad s \geq s_{\min } .
$$

which is based on large- $N_{c}$ and Regge considerations [ए5]-[ए8]. For this reason we will refer to the method of Ref. [R] as the "DV model" and to the method of Ref. [2] as the "truncated-OPE (TOPE) model" . Notice that the truncated OPE model makes the choice $\delta_{V / A}=\infty$ from the outset.

It turns out that the DV model allows us to avoid the above OPE truncation, making a selfconsistent fit possible in a window $s_{\min } \leq s_{0} \leq m_{\tau}^{2}$, in which $\alpha_{s}\left(m_{\tau}\right), C_{6,8}{ }^{2}$ and the $8 \mathrm{DV}$ parameters $\delta_{V / A}, \gamma_{V / A}, \alpha_{V / A}, \beta_{V / A}$ are determined from the data. The fits also determine $s_{\min } \simeq 1.55 \mathrm{GeV}^{2}$ as an optimal choice. Consistent results were obtained in Ref. [2] from a series of fits of this type. However, the results obtained for $\alpha_{s}\left(m_{\tau}\right)$ were systematically $\sim 0.020-0.025$ lower than in the TOPE model. It is important to clarify the underlying cause for this difference.

Two recent analysis [22, [23] have taken up this task. In Ref. [22] a very thorough survey of a variety of fits based on the TOPE strategy has been performed checking for the stability of the result with respect to the inclusion of the first higher term of the OPE neglected in the earlier fits. Of course, once a new term is included, the number of degrees of freedom is zero, the value of the fit quality vanishes and one no longer has a real "fit". However, errors can still be propagated, and Ref. [R2] finds results which display a certain stability with respect to this change. This stability is then taken by the authors of Ref. [R2] as a proof for the robustness and reliability of the results.

\footnotetext{
${ }^{2} C_{2}$ is negligible and the weights are deliberately chosen to avoid $C_{4}$, because the presence of this condensate spoils the perturbative convergence [[26].
} 

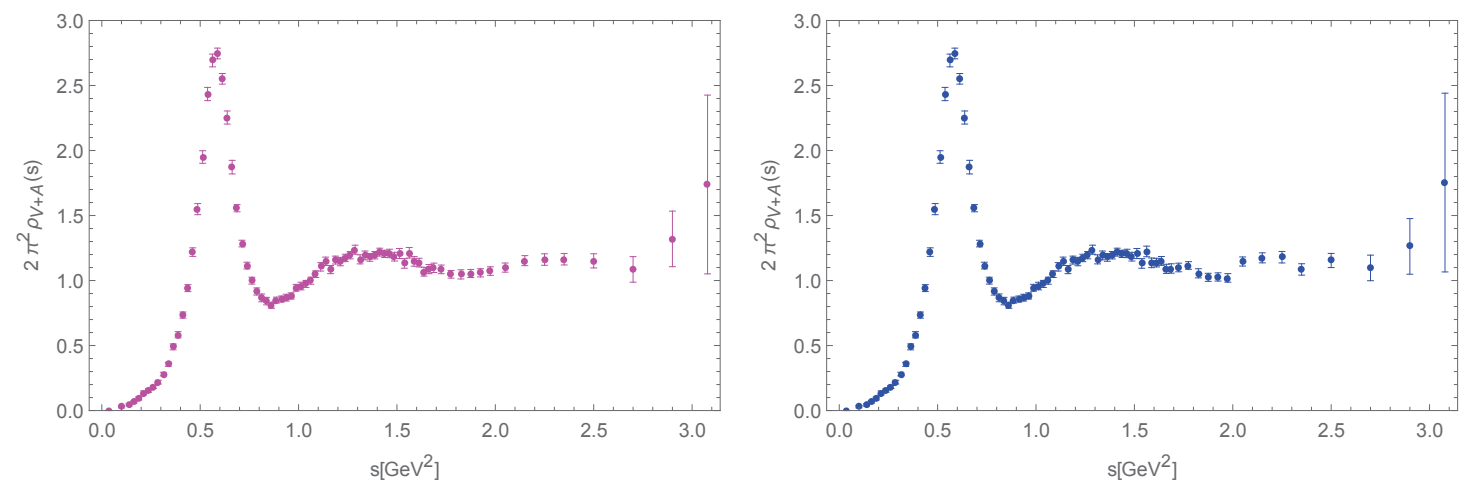

Figure 1: $V+A$ non-strange spectral function. Left panel: fake data, generated as described in the text, as a function of s. Right panel: true ALEPH data [[Z]] as a function of s. The fake data have been generated for $s \geq 1.55 \mathrm{GeV}^{2}$; below this value the two data sets are the same.

It is important to realize, however, that this condition of stability is a necessary, but not sufficient one. It is easily demonstrated that other choices for the higher dimension $C_{D}$ exist which are reasonable from the point of view of what one could expect in $\mathrm{QCD}$, but which produce a different result for $\alpha_{s}\left(m_{\tau}\right)$, one which is equally stable when tested as above [23]. This means the values assumed for the higher $C_{D}$ affect in a significant way the $\alpha_{s}\left(m_{\tau}\right)$ extracted from fits using the TOPE model strategy. The tests conducted in Ref. [22] are thus inconclusive.

Is there a test that discriminates between the DV model strategy and the TOPE model strategy for determining $\alpha_{s}\left(m_{\tau}\right)$ ? The answer is yes. It consists of fitting a set of fake data for the $V+A$ spectral function ${ }^{3}$ constructed with a known value for $\alpha_{s}\left(m_{\tau}\right)(=0.312$, using CIPT), and the DV parametrization in Eq. (D) ${ }^{4}$ by generating a multivariate gaussian distribution at the ALEPH bin energies with central values given by the above parameters and with fluctuations controlled by the real-data covariance matrix. The true and fake $V+A$ data are compared in Fig. 1. They look remarkably similar.

If the TOPE model strategy fails to find the correct model value for $\alpha_{s}\left(m_{\tau}\right)$, it will have been shown to be unreliable and hence not safely usable for the real data either. This is in fact what has been found [23]. The TOPE model strategy extracts a value, $\alpha_{s}\left(m_{\tau}\right) \simeq 0.334(4)$, many standard deviations away from the correct model result, 0.312 . The DV model strategy, on the other hand, can easily reproduce the right value of $\alpha_{s}\left(m_{\tau}\right)$.

One could remark that it was easy for the DV model to get the right answer because it was the DV model that was used as the basis for the construction of the fake data. While this is of course true, the above exercise shows clearly that, by declaring DVs and the high-dimension $C_{D}$ to vanish, the TOPE model leaves itself in general unable to extract the correct value of $\alpha_{s}\left(m_{\tau}\right)$, making it unreliable. The ALEPH data could have sizeable DVs and the TOPE model strategy would never detect them. On the other hand, the DV model is able to detect the amount of DVs through the parametrization (D).

\footnotetext{
${ }^{3}$ According to common lore, this is the combination most favorable for the TOPE model.

${ }^{4}$ The precise values for the parameters can be found in Ref. [22]].
} 


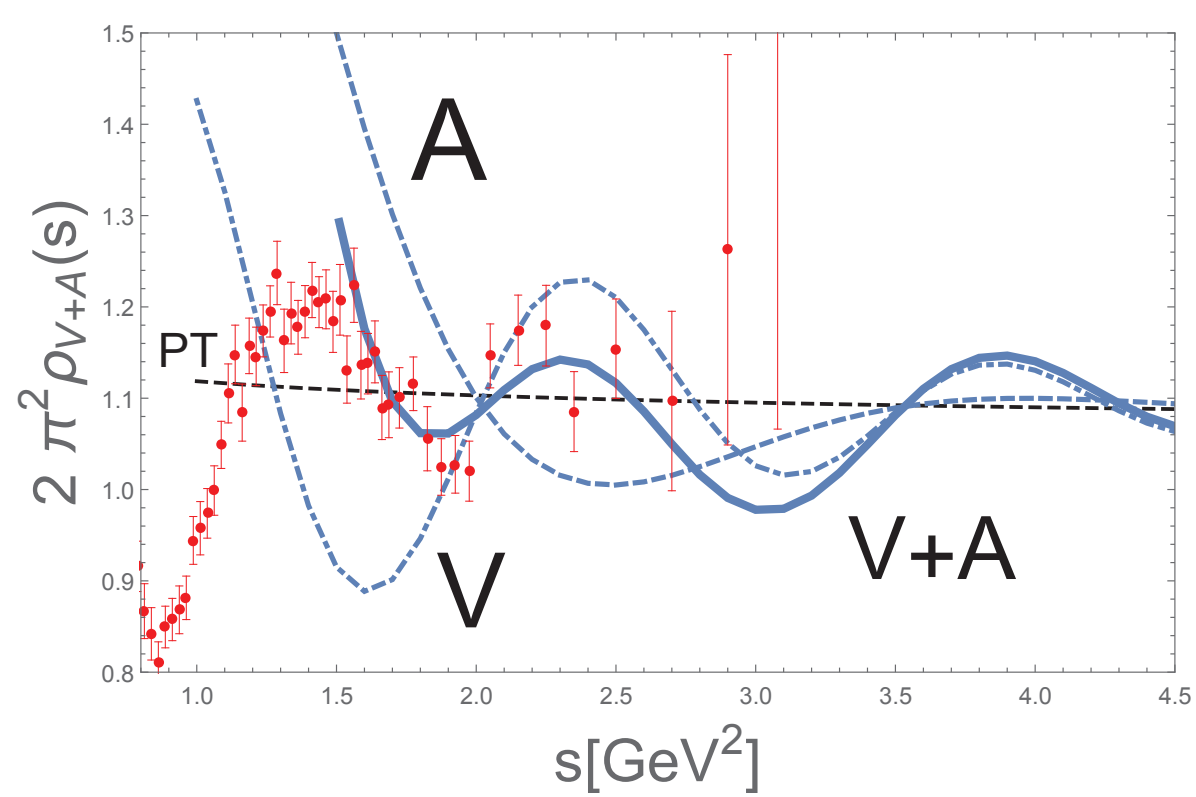

Figure 2: Blow-up of the large-s region of the $V+A$ non-strange spectral function. Black dashed line: the perturbative (CIPT) representation of the model. Blue curve: full model representation, including DVs. Blue dot-dashed curves: separate $V$ and A parts of the model spectral function.

Based on our DV model fits to the ALEPH data, are the DVs large or small? Fig. 2 shows the $V+A$ ALEPH data (red points) together with our DV model fit (solid blue line), the perturbation theory curve (in CIPT, black dashed line) and the corresponding model fits for $V$ (blue dot-dashed curve) and $A$ (blue dashed curve). As one can see, the oscillations in the data points below $s=$ $2.3 \mathrm{GeV}^{2}$ are not small and show no sign of a strong damping. Above this value the data errors are too large to tell. Notice that the difference between the black dashed line (perturbation theory) and an horizontal line at $2 \pi^{2} \rho_{V+A}(s)=1$ (the parton model) represents the dynamical contribution from which the value of $\alpha_{s}\left(m_{\tau}\right)$ is extracted in QCD. This difference is in no way small relative to the oscillations depicted by the blue solid line (DVs) which, by the way, in the region above $s \gtrsim 1.7 \mathrm{GeV}^{2}$, happen to be largest precisely at $m_{\tau}^{2}$, where the TOPE model declares them to be zero.

In summary, the analysis of Ref. [23] shows the TOPE model strategy is unreliable and, consequently, should not be used to determine $\alpha_{s}\left(m_{\tau}\right)$. The DV model strategy, on the other hand, passes all the tests known up to date ${ }^{5}$ and obtains the following values from the ALEPH data [23]]:

$$
\begin{array}{ll}
\alpha_{s}\left(m_{\tau}\right)=0.296(10) & (\mathrm{FOPT}) \\
\alpha_{s}\left(m_{\tau}\right)=0.310(14) & (\mathrm{CIPT})
\end{array}
$$

\footnotetext{
${ }^{5}$ For a detailed refutation of the criticism of our approach in Ref. [22], we refer to Sec. V.B of Ref. [D23].
} 
The work of MG is supported by the U.S. Department of Energy, Office of Science, Office of High Energy Physics, DE-FG03-92ER40711. The work of DB is supported by the São Paulo Research Foundation(FAPESP) Grant No. 2015/20689-9 and by CNPq Grant No. 305431/2015-3. KM is supported by the Natural Sciences and Engineering Research Council of Canada. SP is supported by CICYTFEDER-FPA2014-55613-P, 2014-SGR-1450.

\section{References}

[1] E. Braaten, S. Narison and A. Pich, Nucl. Phys. B 373 (1992) 581.

[2] F. Le Diberder and A. Pich, Phys. Lett. B 289 (1992) 165; Phys. Lett. B 286 (1992) 147.

[3] R. Shankar, Phys. Rev. D 15, 755 (1977).

[4] R. G. Moorhouse, M. R. Pennington and G. G. Ross, Nucl. Phys. B 124, 285 (1977).

[5] K. G. Chetyrkin and N. V. Krasnikov, Nucl. Phys. B 119, 174 (1977).

[6] K. G. Chetyrkin, N. V. Krasnikov and A. N. Tavkhelidze, Phys. Lett. B 76, 83 (1978).

[7] N. V. Krasnikov, A. A. Pivovarov and N. N. Tavkhelidze, Z. Phys. C 19, 301 (1983).

[8] E. G. Floratos, S. Narison and E. de Rafael, Nucl. Phys. B 155, 115 (1979).

[9] R. A. Bertlmann, G. Launer and E. de Rafael, Nucl. Phys. B 250, 61 (1985).

[10] E. Braaten, Phys. Rev. Lett. 60, 1606 (1988).

[11] K. Ackerstaff et al. [OPAL Collaboration], Eur. Phys. J. C 7 (1999) 571, and references therein.

[12] S. Schael et al. [ALEPH Collaboration], Phys. Rept. 421, 191 (2005), and references therein.

[13] M. Davier, A. Hoecker, B. Malaescu, C. Z. Yuan and Z. Zhang, Eur. Phys. J. C 74, 2803 (2014).

[14] D. R. Boito, O. Catà, M. Golterman, M. Jamin, K. Maltman, J. Osborne and S. Peris, Nucl. Phys. Proc. Suppl. 218, 104 (2011).

[15] B. Blok, M. A. Shifman and D. X. Zhang, Phys. Rev. D 57, 2691 (1998) [Erratum-ibid. D 59, 019901 (1999)].

[16] O. Catà, M. Golterman and S. Peris, JHEP 0508, 076 (2005).

[17] O. Catà, M. Golterman and S. Peris, Phys. Rev. D 77, 093006 (2008).

[18] O. Catà, M. Golterman and S. Peris, Phys. Rev. D 79, 053002 (2009).

[19] D. Boito, O. Catà, M. Golterman, M. Jamin, K. Maltman, J. Osborne and S. Peris, Phys. Rev. D 84, 113006 (2011).

[20] D. Boito, M. Golterman, M. Jamin, A. Mahdavi, K. Maltman, J. Osborne and S. Peris, Phys. Rev. D 85, 093015 (2012).

[21] D. Boito, M. Golterman, K. Maltman, J. Osborne and S. Peris, Phys. Rev. D 91, no. 3, 034003 (2015).

[22] A. Pich and A. Rodríguez-Sánchez, Phys. Rev. D 94, no. 3, 034027 (2016).

[23] D. Boito, M. Golterman, K. Maltman and S. Peris, arXiv:1611.03457 [hep-ph].

[24] O. Catà, M. Golterman, S. Peris, Phys. Rev. D 79, 053002 (2009).

[25] S. Peris, D. Boito, M. Golterman and K. Maltman, Mod. Phys. Lett. A 31, no. 30, 1630031 (2016).

[26] M. Beneke, D. Boito and M. Jamin, JHEP 1301, 125 (2013). 